\title{
Photovoltaic Principles and Organic Solar Cells
}

\author{
Peter Würfel ${ }^{\star}$
}

\begin{abstract}
The fundamentals of photovoltaics are reviewed. The necessary requirements for material properties are discussed. Achieving high efficiency with organic solar cells is known to be difficult for two main reasons. Photon absorption does not directly lead to free charge carriers but to excitons with large binding energies which are difficult to dissociate. These excitons have diffusion lengths much smaller than the penetration depth of the incident light, making them difficult to process before their recombination. Ways are discussed how these shortcomings may be overcome. In principle, organic solar cells can be as efficient as inorganic solar cells if the right combination of materials can be found.
\end{abstract}

Keywords: Organic solar cells · Photovoltaics

\section{Introduction}

Organic semiconductors have several disadvantages when employed in solar cells:

i) Photon absorption does not directly generate free charge carriers, but excitons with typical binding energies of several hundred meV. Exciton dissociation has been observed to occur at heterojunction interfaces, but was also observed to be accompanied by substantial energy losses.

ii) The diffusion lengths of excitons and of free carriers are typically in the $\mathrm{nm}$ range and thus much smaller than the penetration depth of the photons. In a planar configuration an organic absorber in a solar cell would have to be much thicker than excitons or free carriers could travel and most of the excitations would be lost by recombination.
${ }^{*}$ Correspondence: Prof. P. Würfel Institut für Angewandte Physik

Universität Karlsruhe (TH)

Wolfgang-Gaede-Str. 1

D-76128 Karlsruhe, Germany

E-Mail: peter.wuerfel@phys.uni-karlsruhe.de
Compared with inorganic solar cells, where these problems do not exist, it seems doubtful that organic solar cells will ever be efficient. However, we have to look more closely at the principles of photovoltaics in order to decide whether these shortcomings of organic materials are a fundamental limitation or whether there are ways to overcome these drawbacks, at least in principle.

\section{Principles of Photovoltaics}

\section{First Step: Conversion of Solar Heat into Chemical Energy}

Solar energy which is to be converted into electrical energy in a solar cell is heat from the sun with a temperature of $\mathrm{T}_{\mathrm{S}}=$ $5800 \mathrm{~K}$. This high temperature follows from the intensity of solar radiation per solid angle and per photon energy interval. When part or all of this radiation is absorbed by a material, excitations are produced at a high density and with the same energy spectrum. Before any interaction with lattice or molecular vibrations occurs, the excitations have the same high temperature $\mathrm{T}_{\mathrm{S}}$ as the photons from the sun. By generating excitations (excitons, electron-hole pairs), heat from the sun has simply been transferred into heat of these excitations.

As in any other heat engine, the working medium, the excitations in our case, has to be cooled down. If this is done reversibly, i.e. without generating additional entropy, the same amount of entropy that is absorbed with the solar energy can be discharged into a heat sink at a lower temperature $\mathrm{T}_{0}$, thereby creating free energy. Because of this lower temperature less energy is lost with the entropy than is absorbed, approximately a fraction $\mathrm{T}_{0} / \mathrm{T}_{\mathrm{S}}$ of the absorbed energy. The remaining energy is then free of entropy and is $\left(1-\mathrm{T}_{0} / \mathrm{T}_{\mathrm{S}}\right)$ of the absorbed energy.

The heat engine working in an absorber of solar radiation comes into operation by the interactions of the excitations with the vibrations. This will, in typically less than a picosecond, cool the excitations down to the temperature $T_{0}$ of the vibrations. In all broad band absorbers this cooling of the excitations reduces their energy, making the energy spectrum of the excitations much narrower, thereby demonstrating that they are now at the low temperature $\mathrm{T}_{0}$. However, a loss of energy of the excitations during the cooling process is an irreversible process. This energy loss is directly transformed into low temperature heat of the vibrations.

Fig. 1 shows an example of the conversion of solar heat into free energy of electron-hole pairs. Since the cooling leaves the high concentrations of electrons and holes unchanged, Fermi-distributions are established with different Fermi-energies for the upper and the lower band. The difference of these new quasi-Fermi-energies is the free energy per electron-hole pair which is obtained from solar energy. In the case of excitons as the primary excitations, we can look at the free electrons and holes which are in thermal equilibrium with the excitons and at their two quasi-Fermi distributions. The difference of their quasiFermi-energies is then equal to the chemical potential of the excitons. 


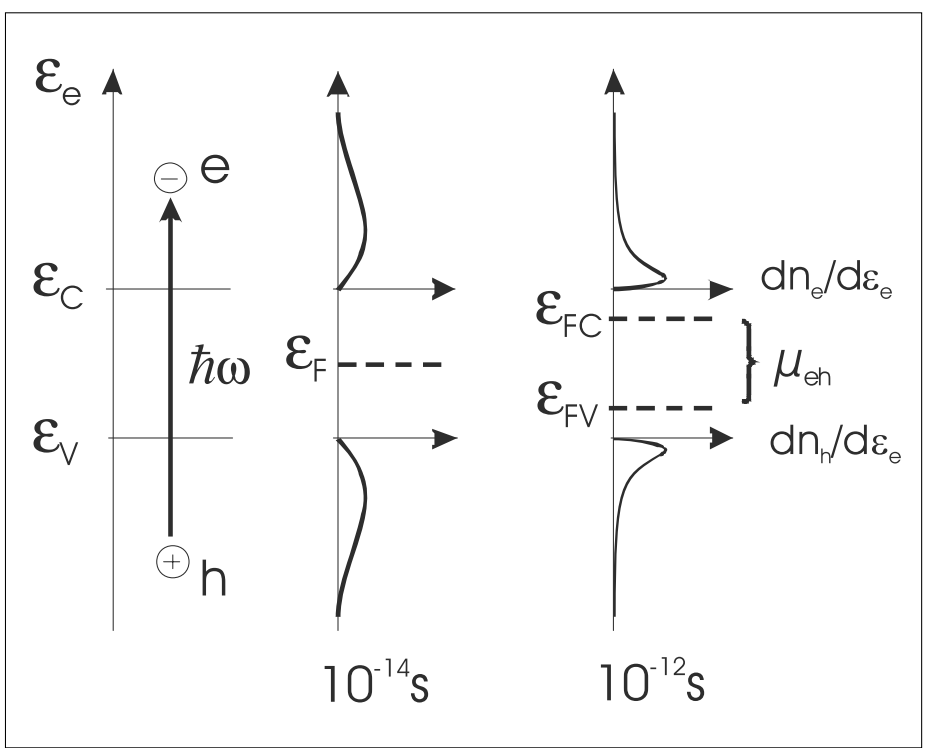

Fig. 1. Conversion of solar heat into chemical energy. By absorption of photons from the sun, a hot electron-hole gas exists up to $10^{-14} \mathrm{~s}$ before scattering with phonons occurs. After about $10^{-12} \mathrm{~s}$ the electron-hole gas is cooled to room temperature. Since the electron and hole concentrations remain unchanged during the cooling process, two separate Fermidistributions with Fermi-energies $\varepsilon_{\mathrm{FC}}$ and $\varepsilon_{\mathrm{FV}}$ evolve. The cooling process converts solar heat $h \omega$ into chemical energy $\mu_{\text {eh }}$ of electron-hole pairs.

In the example in Fig. 1, energy of the electron-hole pairs is lost in the cooling process. How this loss can be avoided is obvious from Fig. 1. The broad band absorber must be replaced by a narrow band absorber. In a narrow band, the energy spectrum of the excitations, electron-hole pairs in the example of Fig. 1, cannot change very much by the cooling process. In the limit of a monochromatic absorber, a loss of energy by the cooling process is completely avoided. It is clear, however, that many different narrow band absorbers are required to absorb all or most of the photons from the broad solar spectrum. Tandem cells operate according to this principle.

Independent of whether electron-hole pairs or excitons are generated, cooling of the primary excitations generates chemical energy, free of entropy. It is this step which is limited by thermodynamics. How much chemical energy is obtained depends on how large the concentration of the excitations is in steady state and this depends on the probability for recombination. Recombination processes can be either radiative or non-radiative and both processes occur in parallel. In a non-radiative recombination process, the energy of the excitation is transferred to vibrations (phonons). To transfer all of the energy of the excitation in one step, a vibration with a very large amplitude (many phonons) must be generated, a process which has a small probability. A non-radiative process is much more likely to occur if the excitation energy is transferred in little amounts in several steps. This requires intermediate states for the ex- citations between the primary excited state and the ground state. Such states are often caused by impurities and can in principle be avoided. Non-radiative recombination is then less probable. That leaves radiative recombination as the major recombination process. Can this be avoided? The answer is no. If a radiative upward transition to generate the excitation is allowed, its reversal, the radiative downward transition must be allowed as well.

Quantitatively, the generation rate $d G_{e h}$ of excitations by absorbing photons with energy $\hbar \omega$ from a black-body at temperature $\mathrm{T}_{\mathrm{S}}$ (the sun) which is seen in a solid angle $\Omega$ is

$$
d G_{e h}=\alpha(\hbar \omega) \frac{\Omega}{4 \pi^{3} \hbar^{3} c^{2}} \frac{(\hbar \omega)^{2}}{\exp \left(\frac{\hbar \omega}{k T_{S}}\right)-1} d \hbar \omega
$$

where $\alpha(\hbar \omega)$ is the absorption coefficient.

The radiative recombination rate $d R_{e h}$ at the same energy $\hbar \omega$ is: ${ }^{[1]}$

$$
\begin{aligned}
d R_{e h}= & \alpha(\hbar \omega) \frac{\Omega}{4 \pi^{3} \hbar^{3} c^{2}} \\
& \frac{(\hbar \omega)^{2}}{\exp \left(\frac{\hbar \omega-\left(\varepsilon_{F C}-\varepsilon_{F V}\right)}{k T_{0}}\right)-1} d \hbar \omega
\end{aligned}
$$

If, as in a monochromatic absorber, generation and recombination occur in the same photon energy interval and in the same solid angle and if there is no non-radiative recombination, then under open circuit con- ditions, both rates must be equal and from Eqns. (1) and (2) we find

$$
\varepsilon_{F C}-\varepsilon_{F V}=\hbar \omega\left(1-\frac{T_{0}}{T_{S}}\right)
$$

The Carnot-factor in Eqn. (3) proves that under the conditions of monochromatic operation and radiative recombination, the conversion of solar heat into chemical energy of electron-hole pairs or excitons is reversible and occurs at the highest possible efficiency. It cannot be improved.

\section{Second Step: Transformation of Chemical Energy into Electrical Energy}

Since chemical energy and electrical energy are both free of entropy the transformation of one form into the other is not restricted by thermodynamics and can, in principle, be $100 \%$ efficient.

For electrical energy, we need a current of charged particles. If the primary excitation is an exciton, then the electron and the hole bound to each other in the exciton must first be separated. We will come to this point later. If the primary excitation consists of a mobile electron and a mobile hole, they only have to move in opposite directions to generate an electrical current or charge current. Such a directional movement is imposed on the electrons and holes in the absorber, if the electrons are able to leave the absorber only on one side and the holes on the other side. The contacts to the absorber must have the properties of semi-permeable membranes allowing the passage of one type of carriers and blocking the other type. A realisation is shown in Fig. 2, where a barrier in the valence band on one side blocks the holes and a barrier in the conduction band on the other side blocks the electrons. These variations in the conduction band and valence band energies are not caused by variations of the electrical potential $\varphi$ but by differences of the electron affinities $\chi_{\mathrm{e}}$ and the band gaps $\varepsilon_{\mathrm{C}}-\varepsilon_{\mathrm{V}}$.

If all electrons move to one side and all holes to the other side then all the charge carriers which are generated and do not recombine contribute to the charge current, which is given by the difference of the generation rate and recombination rate integrated over the thickness of the absorber.

$$
j_{Q}=q \int\left(d G_{e h}-d R_{e h}\right) d x
$$

Eqn. (4), in which the recombination rate depends on the difference of the quasi- 


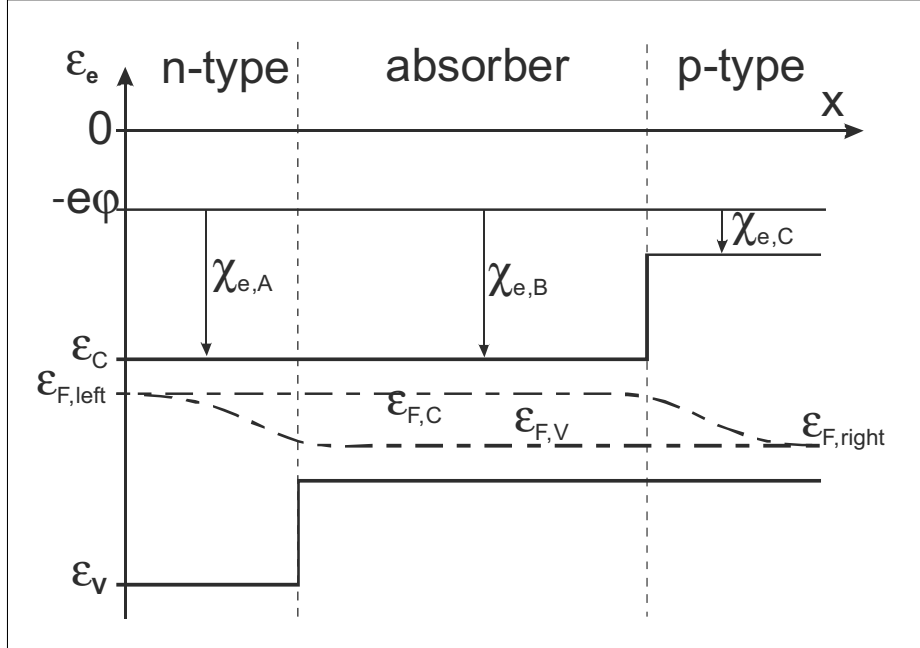

Fig. 2. In an absorber electrons and holes are generated by photon absorption. Due to an energy barrier at the interface with the material on the right, resulting from different electron affinities $\chi_{e}$, electrons can leave the absorber only to the left. Holes can leave only to the right, because of the hole-barrier at the left interface resulting from different band gaps. Small gradients of the quasi-Fermi-energies $\varepsilon_{\mathrm{F}, \mathrm{C}}$ and $\varepsilon_{\mathrm{F}, \mathrm{V}}$ driving electrons to the left and holes to the right cause an electrical current.

Fermi-energies in Eqn. (2), can be seen as the current voltage characteristic for an ideal configuration in which the gradients of the respective quasi-Fermi-energies necessary to drive the electron and hole currents are negligibly small because of large mobilities. In this case the voltage is simply given by

$$
V=\frac{\varepsilon_{F C}-\varepsilon_{F V}}{q}
$$

In conclusion to this simplified and idealized derivation of the function and necessary properties of a solar cell we must keep in mind: A solar cell consists of an absorber and of selective contacts for electrons and for holes, respectively. The recombination rate should be as small as possible, which means that non-radiative recombination should be absent. Nothing can be done to reduce the radiative recombination rate without reducing the absorption rate or the output voltage. A more extensive discussion of the principles of photovoltaics is found in the literature. ${ }^{[1]}$ The most important implication of Eqns. (4) and (5) are that electrons and holes must be able to reach the appropriate selective contact before they recombine. The condition of a high mobility can more easily be seen as a condition for a large diffusion length $L$, which must be larger than the distance between the membranes. On the other hand the thickness of the absorber must be larger than the penetration depth $1 / \alpha(\hbar \omega)$ of the absorbable photons.

How do organic semiconductors fit into this set of conditions?

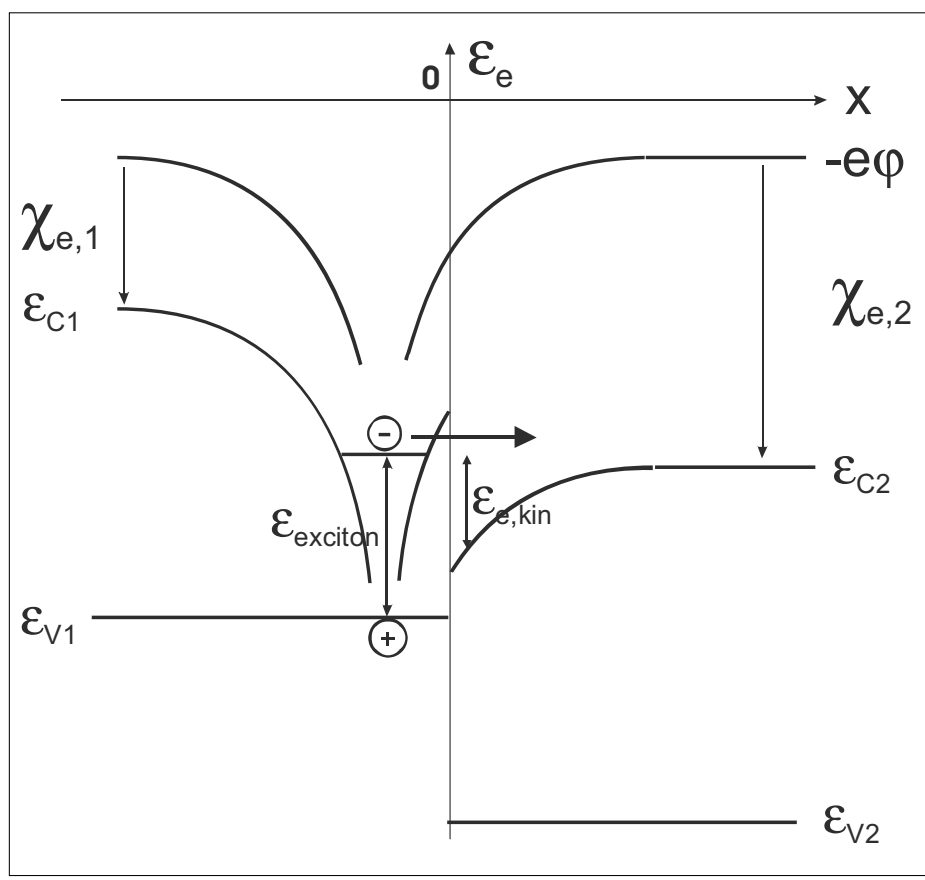

Fig. 3. An electron bound to a hole in an exciton in the material on the left is able to tunnel from its bound state into a free state in the material on the right, where it has enough kinetic energy $\varepsilon_{\text {e,kin }}$ to leave the attraction of the hole, which is then free to move in the material on the left. The lower energy of a conduction state $\varepsilon_{\mathrm{C} 2}$ on the right follows from a larger value of the electron affinity $\chi_{\mathrm{e} 2}$.

\section{Organic Semiconductors}

In organic materials electrons are rather localised. This results in large effective masses, small mobilities and small diffusion coefficients. ${ }^{[2]}$ Optical excitation leads to excitons with binding energies much larger than $k T$. These excitons are stable against thermal ionisation at room temperature. In addition electron states exist for free electrons which are more extended. They allow transport over large distances. Following semiconductor terminology, we call these states conduction states with a lower energy of $\varepsilon_{\mathrm{C}}$, these states may also be called LUMO (lowest unoccupied molecular orbital). Similarly conduction states for holes have an upper energy (on an electron scale) of $\varepsilon_{\mathrm{v}}$, also called HOMO (highest occupied molecular orbital). ${ }^{[3]}$ Like inorganic semiconductors, many organic materials can be doped n-type or p-type. ${ }^{[4]}$

\section{Light Absorption and Recombination}

Organic materials with large absorption coefficients in the order of $105 / \mathrm{cm}$ are quite common. For nearly complete absorption of the incident light, a thickness of the absorber of some tenth of $1 \mu \mathrm{m}$ is sufficient. Nevertheless, this is much larger than the diffusion length of excitons and they will not be able to traverse the absorber. With regard to recombination, organic materials are rather favourable. It is not uncommon to find materials with a high fluorescent yield, which means that almost all of the recombination is radiative. It can be expected that the conversion of solar heat into chemical energy of excitons is rather ideal. In this respect organic materials have an advantage over inorganic materials where non-radiative recombination predominates. Organic materials are also favourable for use in tandem structures with reduced energy loss by thermalisation, where a narrow absorption spectrum is no disadvantage.

\section{Dissociation of Excitons into Electrons and Holes}

For an electrical current the transport of electrons and holes is necessary. In the excitons generated by photon absorption in organic materials, electrons and holes are strongly bound. Typical binding energies of some $100 \mathrm{meV}$ are much too large for the excitons to be ionised thermally at room temperature. Since no energy supply is available to provide for the binding energy, free electrons and holes must be generated from excitons without additional energy. It is known that this may happen at interfaces between different materials. ${ }^{[3]}$ Fig. 3 elaborates how this may be achieved.

Since this Fig. is a diagram of the energy of electrons, the energy $\varepsilon_{\text {exciton }}$ of an exciton as the sum of the energies of a bound electron and hole is seen as an energy difference. By regarding the exciton energy as the energy of a bound electron with respect 
to a free hole it is counted in the Figure from the $\varepsilon_{\mathrm{v} 1}$ level upwards. The energy of the bound electron is then smaller by the exciton binding energy than the energy of a free electron in a $\varepsilon_{\mathrm{C} 1}$ level. (In the same way the exciton energy could be seen as the energy of a bound hole with respect to a free electron and would then be counted from the $\varepsilon_{\mathrm{C}}$ level downwards.) The absorber on the left in Fig. 3 is in contact with a second material in which the energy of a free electron, the $\varepsilon_{\mathrm{C} 2}$ level, is more negative caused by a larger electron affinity $\chi_{\mathrm{e} 2}$. Far away from the hole, the electron in this $\varepsilon_{\mathrm{C} 2}$ level has energy less or equal to the energy of the bound electron in the left material, the absorber. This enables the electron to tunnel from its bound state in the absorber into a free state in the adjacent material which we therefore call an electron collector. However, even in a free state in the electron collector, the electron would experience the Coulomb attraction of the hole. The variation of the electrical potential energy $-\mathrm{e} \varphi$ for this Coulomb interaction extends over both materials. Due to a larger electron affinity $\chi_{\mathrm{e} 2}$ in the electron collector, its $\varepsilon_{\mathrm{C} 2}$ level is down-shifted by the difference of the electron affinities of the two materials. Since in the electron collector in the vicinity of the interface, the energy of the electron is larger than its potential energy, it has enough kinetic energy to leave the attraction of the hole and be really free. In this state of separated electrons and holes the sum of the energies of electron and hole may be equal or a little less than the exciton energy, so that little energy is lost in the configuration of Fig. 3 in generating free electrons and holes from their bound state in an exciton. To avoid energy losses in this process, the two materials must have electron affinities which differ by little more than the exciton binding energy. ${ }^{[3]}$

A problem occurs if the electron loses part of its kinetic energy by scattering while it is still attracted by the hole. It will then not be able to leave the hole and both will be trapped at the interface where they will eventually recombine. To guard against this trapping, the $\varepsilon_{\mathrm{C}}$ level in the electron collector should be somewhat lower (50-100 meV) than the electron energy in the exciton in the absorber to allow for some energy loss by scattering without trapping the electron.

The interface between the absorber and this electron collector serves two purposes: It generates free electrons and holes and in addition it separates them locally so that they can no longer recombine. The interface is the required selective contact for electrons and the electron collector must be a good electron conductor and should not provide any states for free holes to go from the absorber into this material. The $\varepsilon_{\mathrm{V} 2}$ level of the electron collector should therefore be some 100 meV below the $\varepsilon_{\mathrm{V} 1}$ level of the absorber. In the configuration of Fig. 3, the absorber must have a good hole conductivity, i.e. it must be p-type because free holes are produced at the interface with the electron collector, from where they have to reach the hole contact. Since this charge transport is then among the majority carriers, it is not limited by a diffusion length. Although the p-type absorber provides selective transport for holes only, with no free electrons around, it should not be in direct contact with a metal electrode, because of the excitons in the absorber which would recombine efficiently at the interface with a metal. This problem is solved by using a p-type hole collector with an $\varepsilon_{\mathrm{V}}$ level close to that of the absorber to remove the holes from the absorber. Since there are no free electrons in the absorber, no requirements exist on the energetic position of the $\varepsilon_{\mathrm{C}}$ level of the hole collector with respect to electron transfer. However, the incident sun light must not be absorbed in the collector layers, requiring a difference $\varepsilon_{\mathrm{C}}-\varepsilon_{\mathrm{V}}$ of the collector layers of approximately $3 \mathrm{eV}$. In addition the charge carrier collectors must be well conducting materials to avoid voltage losses. ${ }^{[4]}$

If the absorber is an n-type material, everything is reversed. Exciton ionisation must then occur at the interface with the hole collector, which must have $\varepsilon_{\mathrm{V}}$ states for free holes with energies on the electron energy scale of Fig. 3 higher by a little more than the exciton binding energy of the $\varepsilon_{\mathrm{V}}$ states of the absorber. The electron collector on the other side should have states close to the energy of the $\varepsilon_{\mathrm{C}}$ states of the absorber to allow for easy electron transfer. Both electron and hole collectors, for which the term transport layers is also used, must be well conducting.
Using both interfaces of the absorber with the electron collector and with the hole collector for exciton ionisation is not a good idea. It would lead to free electrons and free holes in the absorber which could recombine. Their transport would therefore be limited by their diffusion lengths. In addition, an energy loss of the amount of the exciton binding energy would occur per exciton, since the electron and the hole collector would both have to accept carriers which are free in the absorber as well as carriers which are bound to excitons in the absorber which, after tunnelling into the appropriate collector, have energies smaller by the exciton binding energy. The higher energy of the carriers which were free in the absorber would be lost by thermalisation in the collector.

There is a problem for the transport of the excitons. They are generated by photon absorption throughout the absorber and they all have to move towards the interface with the electron collector which must therefore be within a diffusion length of their point of generation. Since the diffusion length of excitons is typically in the nm range and much smaller than the penetration depth $1 / \alpha$ of the photons in the absorber, efficient exciton transport and sufficient absorption seem to be incompatible.

\section{Efficient Exciton Transport with Small Diffusion Lengths}

For efficient exciton transport, the distance between the electron and the hole collector must be smaller than an exciton diffusion length. In the planar configuration of Fig. 4a, this leads to an absorber which is too thin to absorb sufficient light. Fold-

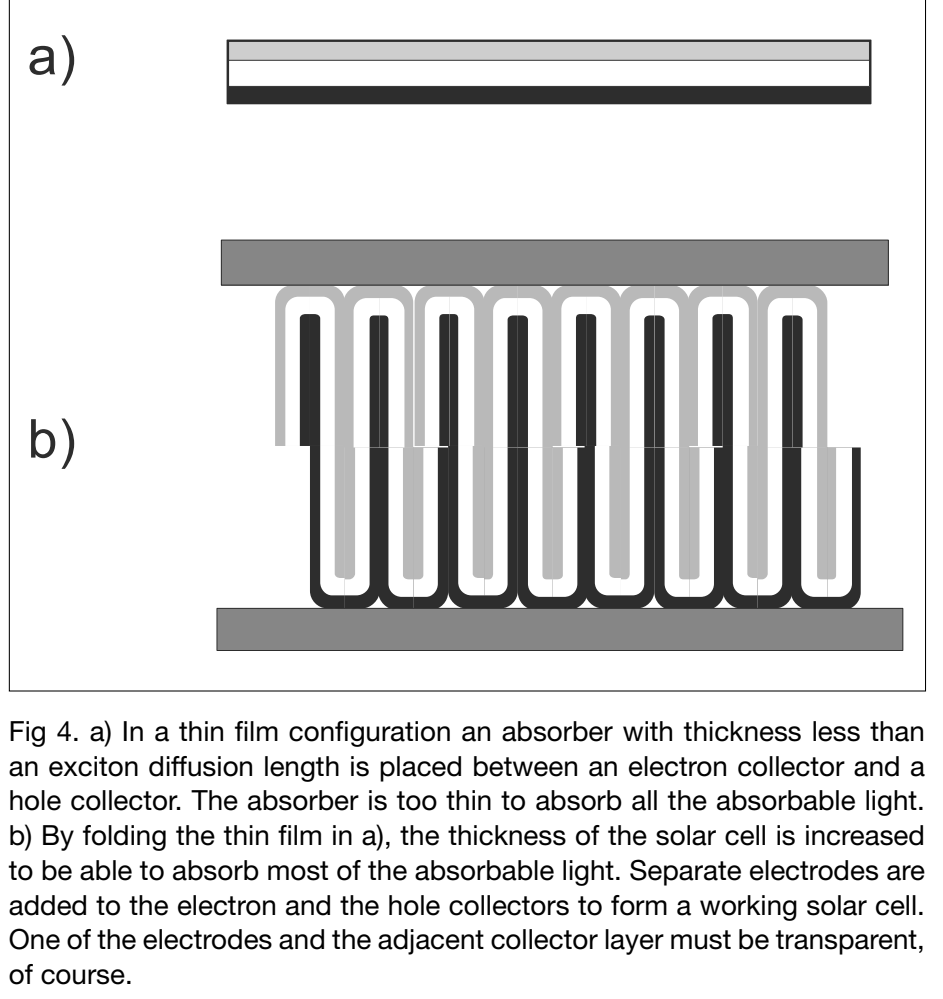


ing the planar configuration as in Fig. $4 \mathrm{~b}$ increases the probability for absorption but leaves the condition for efficient exciton transport unchanged. In this way, small diffusion length and large absorber thickness can be made compatible. For typical properties of organic materials, the effective thickness of the absorber must be between ten and hundred times as large as the distance between the charge carrier collectors.

\section{Conclusions}

We have described an ideal configuration for organic materials in a solar cell. The apparent disadvantages of organic materials; the generation of strongly bound excitons instead of free electrons and holes and the small exciton diffusion length can be overcome, in principle. The high fluorescent yield of many organic materials demonstrates that non-radiative recombination is less of a problem than in inorganic materials.

The large interface area between absorber and collectors, a consequence of the small exciton diffusion length, would be prohibitive with inorganic materials because of increased non-radiative recombination at interface states. For organic materials with their weak van der Waals bonding, interface states caused by dangling bonds are less of a problem and large interface areas seem to be tolerable.
Existing examples of organic solar cells incorporate the mechanisms described above. The first hetero-junction solar cell where the exciton dissociation at the interface was incorporated in a planar bi-layer configuration was presented by C. Tang. [5] More advanced versions containing electron and hole collector layers are described in ref. [4].

The bulk hetero-junction solar cell is another realisation of the model presented here. The p-type absorber (PPV or similar) is mixed with an electron collector (C60 or similar). ${ }^{[6]}$ As a result, the electron collector may not be everywhere within reach of the excitons or may be in direct contact with the hole collector. Whether the energetic alignment of all the energy levels involved conforms to the model above is questionable. There seems to be room for improvement.

Received: October 12, 2007

[1] P. Würfel, 'Physics of Solar Cells', Wiley $\mathrm{VCH}, 2005$.

[2] B. A. Gregg, S.-G. Chen, R. A. Cormier, Chem. Mater. 2004, 16, 4586.

[3] P. Peumans, S. R. Forrest, Chem. Phys. Lett. 2004, 398, 27.

[4] K. Walzer, B. Maennig, M. Pfeiffer, K. Leo, Chem. Rev. 2007, 107, 1233.

[5] C. Tang, Appl. Phys. Lett. 1986, 48, 183.

[6] S. E. Shaheen, C. J. Brabec, F. Padinger, T. Fromherz, J. C. Hummelen, N. S. Sariciftci, Appl. Phys. Lett. 2001, 78, 841. 